\title{
HiERARCHY OF PERIOdic SOlUtions FOR HAMILTONIAN SYSTEMS AND THEIR APPlications to Celestial Mechanics
}

\author{
Yu.V. Barkin \\ Moscow State Technical University, Chair of Theoretical Mechanics, 52nd \\ Bauman Street, Moscow, Russia
}

\begin{abstract}
A systematic investigation has been carried out for periodic solutions for standard-form Hamiltonian systems containing a small parameter/the principal problem of dynamics/. An efficient method of investigation of conditions for periodicity of solutions has been developed. Besides fitting the initial conditions of the action-angle variables, the idea of fitting the values of the parameters of the problem is used. Constructive conditions are obtained for the existence of periodic solutions in both principal and degenerate cases, as well as necessary conditions for their stab-ility;algoriths have been developed for constructing these solutions as series in integer powers of the amall parameter. To study particular periodic solutions /by high order resonances/, canonical transformations of the initial equations to a special form are used.
\end{abstract}

1. Consider a one-degree nonautonomous Hamiltonian system

$$
\begin{aligned}
& \frac{d I}{d t}=\frac{\partial H}{\partial \psi} \quad \frac{d \psi}{d t}=-\frac{\partial H}{\partial I} \\
& H=H_{O}(I, A, B)+\sum_{\sigma=1}^{\infty} \mu \sigma_{\sigma}(I, \psi, A, B, \Omega, t)
\end{aligned}
$$

Here $\mathrm{H}$ is the unperturbed Hamiltonian being a function of the position variable $I$ and of the problem parameters $A, B$. The Hamiltonian $H$ in (1) is a holomorphous function of a 
small nonnegative parameter $\mu$ in some vicinity of $\mu=0$. The functions $H_{\sigma}(I, \psi, A, B, \Omega t)$ are $2 \pi$ perlodic in the angular variable $\psi$ and $\Omega \mathrm{t}$ is a given fixed frequency, $T=2 \pi / \Omega$ is $t$ he period.

For $\mu=0$ we obtain from (1) a generating periodic solution [1]

$$
\left.I=a, \psi=n^{(0)}\right)_{t+w}, n^{(0)}=-\frac{\partial H_{0}}{\partial a}, q_{0} n^{(0)}\left(a, \alpha_{0}, B_{0}\right)=q \Omega
$$

Here $w$ is an arbitrary constant, $n^{(0)}$ is the unperturbed frequency, $q_{0}$ and $q$ are integer numbers. The generating values of the variable $I$ and of the parameters $A, B$ are designated as $a$ and $\alpha_{0}, \beta_{0}$.

Let $a+\delta$ and $w+\gamma$ be the initial values of the variables, and $A=\alpha_{0}+\alpha, B=\beta_{0}+\beta$ are the parameter values for $t$ he sought periodic solutions. According to the proposed method, the quantities $\delta, \gamma$, and in certain conditions the quantities $\alpha, \beta$ as well, are constructed as series in integer or fractional powers of the small parameter $\mu$. The proof of existence of periodic solutions is basing on the analysis of the periodicity conditions and of their resolvability with respect to some two out of four small quantities $v=(\delta, \gamma, \alpha, \beta)$ [2].

Let us designate as $\overline{\mathbf{f}}$ and $\tilde{\mathrm{f}}$ the constant and the conditionally periodic parts, respectively, of the function $f(I, \psi, A, B, \Omega t)$ being $2 \pi$ periodic in $\psi$ and in $\Omega t$ and calculated for $t$ he generating values of the parameters (2), i.e.

$\bar{f}\left(a, w, \alpha_{0}, \beta_{0}\right)=\frac{1}{T} \int_{0}^{T} f\left(a, n{ }^{(o)} t+w, \alpha_{0}, \beta_{0}, \Omega t\right) d t, \quad \tilde{f}=0$

The main forms of the existence conditions of periodic solutions of the system (1) are obtained as a result of an analysis of the main terms in the expansion of the periodicity conditions in powers of the small quantities $v$.

Besides the condition of commensurability of frequencies $n^{(0)}, \Omega$, conmon here is the condition of $H$. Poincare [1]

$$
\frac{\partial \overline{\mathrm{H}}_{1}}{\partial w}\left(a, w, \alpha_{0}, \beta_{0}\right)=0
$$

to which one of the following four additional conditions must be added: 


$$
\begin{aligned}
& 1.1 \quad \frac{\partial n^{(0)}}{\partial a} \neq 0 \\
& \frac{\partial^{2} \overline{H_{1}}}{\partial w^{2}} \neq 0 \\
& \frac{\partial^{2} \bar{H}_{1}}{\partial w^{2}} \neq 0 \\
& 1.2 \frac{\partial \mathrm{n}(0)}{\partial \alpha_{0}} \neq 0 \\
& \frac{\partial \mathrm{n}^{(0)}}{\partial \mathrm{a}} \frac{\partial^{2} \overline{\mathrm{H}}_{1}}{\partial \alpha_{0}{ }^{\partial w}} \neq 0 \\
& 1.3 \\
& \frac{\partial n}{\partial \alpha_{0}} \frac{\partial^{2}{\overrightarrow{H_{1}}}}{\partial a w^{2}}- \\
& \frac{\partial n^{(0)}}{\partial B_{0}} \frac{\partial^{2} \bar{H}_{1}}{\partial \alpha_{0} \partial w} \neq 0
\end{aligned}
$$

In the conditions 1.2 and 1.3 the parameter $\alpha_{0}$ can be replaced by $B_{0}$.

Note al so that for the periodic solutions determined by the conditions (2), ( 3 ) and $1.2-1.4$ the parameters $A, B$ are constructed as series in integer powers of the small parameter $\mu$.

Let the Hamiltonian system (1) be such that the function does not contain resonant terms, $i, e . \bar{H}_{1}=\bar{H}_{1}(w)$. This situation takes place in many problems of Celestial Mechanics, e.g., in high-order resonances. In this case, as a result of deeper analysis of periodicity conditions, new conditions were obtained for the existence of periodic solutions, generated from the solutions (2). For these groups the common condition is

$$
\frac{\partial \bar{K}}{\partial w}\left(a, w, \alpha_{0}, \beta_{0}\right)=0 \text {, }
$$

which must be supplemented by one of the following four conditions:

2.1

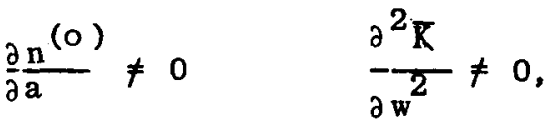

2.2

$$
\frac{\partial n^{(0)}}{\partial \alpha_{0}} \neq 0 \quad \frac{\partial^{2} \bar{K}}{\partial w^{2}} \neq 0 \text {, }
$$

$$
\frac{\partial n(0)}{\partial \alpha_{0}} \frac{\partial^{2} \bar{K}}{\partial a \partial w}-\frac{\partial n^{(0)}}{\partial a} \frac{\partial^{2 \bar{k}}}{\partial \alpha_{0} \partial w} \neq 0
$$


2.4

$$
\frac{\partial n(0)}{\partial \alpha_{o}} \frac{\partial^{2} \bar{K}}{\partial \beta_{o} \partial w}-\frac{\partial n(0)}{\partial \beta_{o}} \frac{\partial^{2} \bar{K}}{\partial \alpha_{o} \partial w} \neq 0
$$

where

$$
\overline{\mathrm{K}}=\frac{\partial^{2} \tilde{\mathrm{H}}_{1}}{\partial \mathrm{a} \mathrm{w}} \tilde{\mathrm{I}}_{1}+\overline{\frac{\partial^{2} \tilde{\mathrm{H}}_{1}}{\partial \mathrm{w}^{2}}} \tilde{\psi}_{1}+\frac{\partial \overline{\mathrm{H}}_{2}}{\partial \mathrm{w}}
$$

and $\mu \tilde{I}_{1}, \mu \tilde{\psi}_{1}$ are purely periodic components of the first-order perturbations in the sought solution.

Of interest is a more general case of degeneracy. When resonant terms appear only in construction of perturbation of a certain arbitrary order $p$. Using the method of canonical transformations/e.g., the method of Deprit-Hri $[3,4,5] /$, the initial differential equations (1) in a sufficiently general case can be reduced to a canonical system with a lamiltonian

$$
\mathrm{H}=\mathrm{H}_{\mathrm{O}}(\mathrm{I}, \mathrm{A}, \mathrm{B})+\sum_{\sigma=\mathrm{X}}^{\mathrm{P}-1} \mu^{\sigma} \mathrm{H}_{\sigma}(\mathrm{I}, \mathrm{A}, \mathrm{B})+\sum_{\sigma=\mathrm{p}}^{\infty} \mu^{\sigma} \mathrm{H}_{\sigma}(\mathrm{I}, \psi, \mathrm{A}, \mathrm{B}, \Omega \mathrm{t})
$$

Periodic solutions of the transformed equations with the Hamiltonian (4) are generated from the solutions (2) at small values of $\mu$ if, besides the conditions

$$
q_{0} n^{(0)}\left(a, \alpha_{0}, \beta_{0}\right)=q \Omega, \quad \frac{\partial \bar{H}_{p}}{\partial w}\left(a, w, \alpha_{0}, \beta_{0}\right)=0
$$

anyone of the following four conditions is fulfilled:

$$
\begin{aligned}
& 3.1 \quad \frac{\partial n^{(0)}}{\partial a} \neq 0, \quad \frac{\partial^{2} \bar{H}_{p}}{\partial w^{2}} \neq 0, \\
& 3.2 \quad \frac{\partial \mathrm{n}(0)}{\partial \alpha_{0}} \neq 0, \quad \frac{\partial^{2} \overline{\mathrm{h}}_{\mathrm{p}}}{\partial \mathrm{w}^{2}} \neq 0, \\
& 3.3 \frac{\partial n}{\partial \alpha_{0}} \frac{\partial^{2} \overline{\mathrm{H}}_{\mathrm{p}}}{\partial \mathrm{a} \partial \mathrm{w}}-\frac{\partial \mathrm{n}(0)}{\partial \mathrm{a}} \frac{\partial^{2} \overline{\mathrm{H}}_{\mathrm{p}}}{\partial \alpha_{0} \partial \mathrm{w}} \neq 0 \text {, } \\
& 3.4 \frac{\partial n^{(0)}}{\partial \alpha_{0}} \frac{\partial^{2} \bar{H}_{p}}{\partial B_{o} \partial w} \quad \frac{\partial n^{(0)}}{\partial B_{o}} \frac{\partial^{2} \bar{H}_{p}}{\partial \alpha_{0} \partial w} \neq 0
\end{aligned}
$$


2. Periodic solutions determined by the conditions 1.1-1.4, $2.1-2.4$ and $3.1-3.4$ are represented by series in integer powers of the small parameter $\mu$ converging by its small value.

As a result of a deeper analysis of the periodicity conditions, periodic solutions of the equations (1) were studied; these solutions can be represented as series in fractional powers of the small parameter $\mu$.

The conditions of existence of periodic solutions which were obtained as a result of an analysis of two and three multiple roots of the corresponding periodicity conditions, can be written down as an ensemble of conditions $\theta_{i}{ }^{(z)}$ and $\phi_{j}(z, u)$ :

$$
\left(\theta_{i}^{(z)}, \phi_{j}^{(z, u)}\right)
$$

$$
\left(z=\left(a, \alpha_{0}, \beta_{0}\right), u=\left(a, w, \alpha_{0}, \beta_{0}\right), \quad z \neq u ; \quad i, j=1,2, \ldots, 15\right)
$$

The conditions $\theta_{i}^{(z)}$, in their turn, are given in Table 1 , and the conditions $\phi_{j}(z, u)$ are obtained from the conditions by a formal substitution of $\psi_{i}$ to $F_{i}^{(z, j)}, i=0,1,2,3,4$, of arguments $z$ to $u$, and of the quantities, in particular, $z_{1}^{(i)}$ to $u_{1}^{(i)}$. Here $z_{1}^{(i)}=\left(\delta_{1}^{(i)}, \alpha_{1}^{(i)}, \beta_{1}^{(i)}\right)$ and $u_{1}^{(i)}=\left(\delta_{1}^{(i)}\right.$, $\left.\gamma_{1}^{(i)}, \alpha_{1}^{(i)}, B_{1}^{(i)}\right)$. The integer exponents $k^{(z)}$, characterizing the multiplicity of the corresponding roots of the periodicity conditions, are replaced by $k(u)$. Some formulae, allowing to describe in detail the structure of the existence conditions (6) are given in the Appendix.

If the conditions (6) are fulfilled, then the differential equations (1) admit periodic solutions which can be represented by series in, generally speaking, fractional powers of the parameter $\mu$ :

$$
\begin{aligned}
& I^{(z, u ; i, j)}=a+\sum_{\zeta=1}^{\infty} \mu^{\frac{\zeta}{(z)} k_{j}^{(u)}} I_{\zeta}^{(z, u ; i, j)}(t) \\
& \psi^{(z, u ; i, j)}=n^{(o)}(a) t+w+\sum_{\zeta=1}^{\infty} \frac{\zeta}{\mu^{(z) k_{j}(u)}} \psi_{\zeta}^{(z, u ; i, j)},
\end{aligned}
$$


Table 1: The conditions $\theta_{j}^{(z)}(j=1,2, \ldots, 15)$

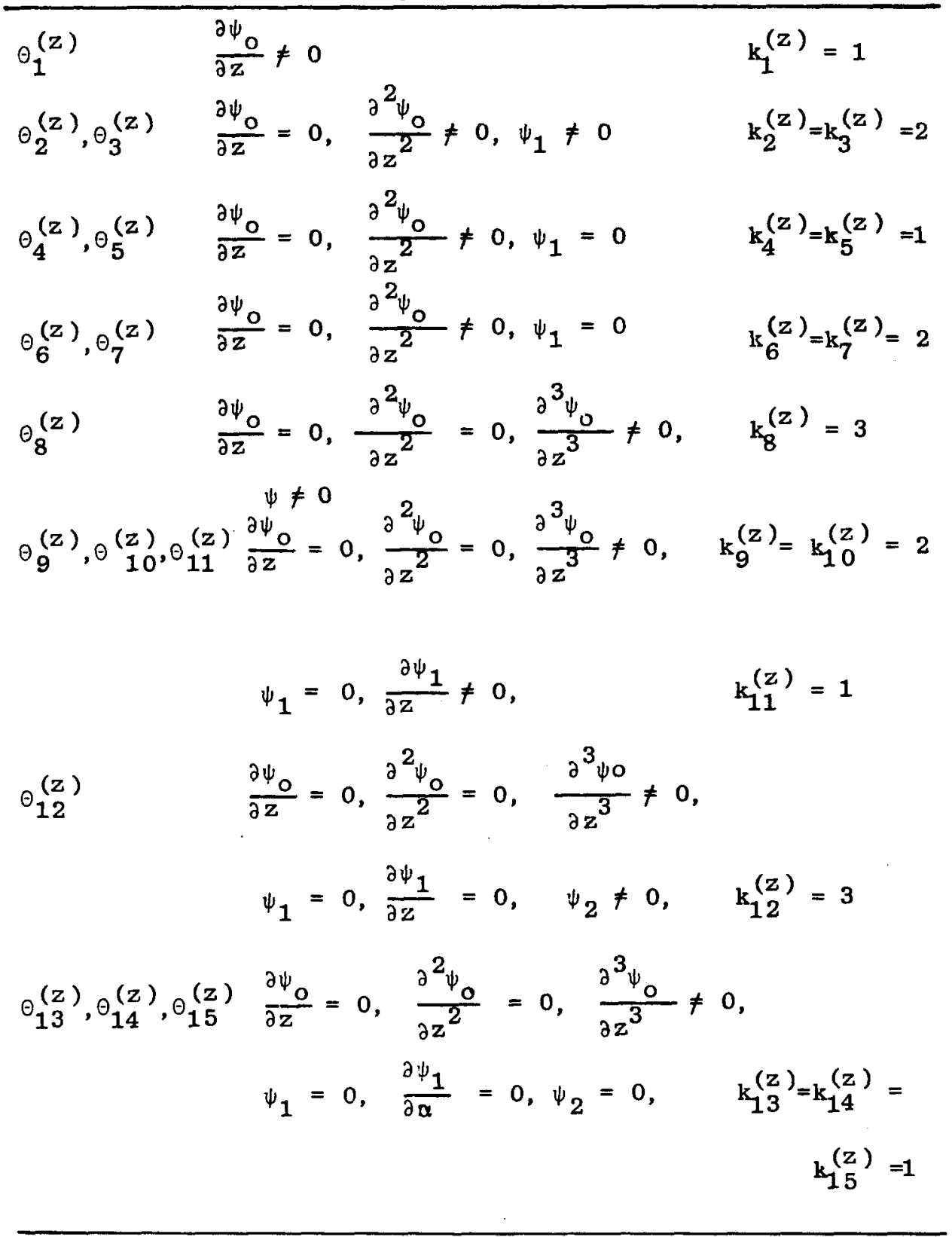


where $I_{\zeta}^{(\ldots)}, \psi_{\zeta}^{(\ldots)}$ are periodic functions of time,determined sequentially by means of simple quadratures.

A list of particular cases of existence of periodic solutions of the Hamiltonian systems (1) can be extended as much as one may like. Derivation of new conditions of existence must base on a detailed analysis of the periodicity conditions and of the conditions of their resolvability. Thereby, as we did earlier, the idea of selection of the problem parameters in form of corresponding series in integer or fractional powers of $\mu$ can be efficiently used.

3. The a bove-mentioned method of investigation of periodic solutions allows to study the existence of periodic solutions in vicinities of fixed points. Here it is advisable to use normal forms of Hamil tonians [5]:

$$
\begin{aligned}
H= & H_{0}(I, A, B)+\mu^{2} H_{2}(I, A, B)+\ldots+\mu^{2 n-2} H_{2 n}(I, A, B)+ \\
& +\mu^{2 n-1} H_{2 n+1}(I, U, A, B, \Omega t)+O\left(\mu^{2 n}\right)
\end{aligned}
$$

for the resonances $(2 n+1) n(0)=m \Omega$,

$$
\begin{aligned}
& \mathrm{H}=\mathrm{H}_{0}(I, A, B)+\mu^{2} \mathrm{H}_{2}(I, A, B)+\ldots+\mu^{2 n-2} \mathrm{H}_{2 n}(I, A, B)+ \\
& +\mu^{2 n} \mathrm{H}_{2 \mathrm{n}+2}(\mathrm{I}, \psi, \mathrm{A}, \mathrm{B}, \Omega \mathrm{t})+O\left(\mu^{2 \mathrm{n}+1}\right)
\end{aligned}
$$

for the resonances $2(n+1) n^{(0)}=m \Omega$ ( $n, m$ are integer numbers).

For the function $\overline{\mathrm{H}}$... we have the following expressions, depending on the type of $\ddot{r}$ esonance

$$
\begin{aligned}
& \overline{\mathrm{H}}_{2 \mathrm{n}+1}=\mathrm{h}_{2 \mathrm{n}+1}\left(\alpha_{0}, \mathrm{~B}_{0}\right) \mathrm{a}^{\mathrm{n}+\frac{1}{2}} \cos (2 n+1) w, \\
& \text { if }(2 n+1) n(0)=m \Omega, \\
& \overline{\mathrm{H}}_{2(n+1)}=\alpha^{n+1}\left[g_{2(n+1)}\left(\alpha_{0}, \beta_{0}\right)+\tau_{2(n+1)}\left(\alpha_{0}, \beta_{0}\right) \cos 2(n+1) w\right], \\
& \text { if } 2(n+1) n(0)=m \Omega,
\end{aligned}
$$

where $h_{2 n+1}, g_{2(n+1)}, \tau_{2(n+1)}$ are constant coefficients of the normal forms. The parameter $\mu$ is introduced into (7), (8) artificially, by a simple substitution of variables. 
The structure of the Hamiltonians (7), (8) corresponds to (4); therefore, for an investigation of periodic solutions here the conditions (5), 3.1 - 3.4 are valid. By means of these conditions we obtain the following four groups of conditions of existence of periodic solutions of canonical equations with the Hamiltonians (7), (8):

$4.1 \frac{\partial n^{(0)}}{\partial \alpha_{0}} \frac{\partial h_{2 n+1}}{\partial \beta_{0}}-\frac{\partial n(0)}{\partial \beta_{0}} \frac{\partial h_{2 n+1}}{\partial \alpha_{0}} \neq 0$

$$
\begin{aligned}
& \alpha \neq 0,(2 n+1) w \neq \quad \pi k, \quad(k=0,1,2, \ldots) \\
& h_{2 n+1}\left(\alpha_{0}, \beta_{0}\right)=0,(2 n+1) n{ }^{(0)}\left(\alpha_{0}, \beta_{0}\right)=m
\end{aligned}
$$

$4.2 \frac{\partial n(0)}{\partial \alpha_{0}} \frac{\partial \tau_{2}(n+1)}{\partial \beta_{0}}-\frac{\partial n}{\partial \beta_{0}} \frac{\partial \tau_{2}(n+1)}{\partial \alpha_{0}} \neq 0$

$\alpha \neq 0, \quad 2(n+1) w \neq \pi k$

$$
\tau_{2(n+1)}\left(\alpha_{0}, \beta_{0}\right)=0,2(n+1) n^{(0)}\left(\alpha_{0}, \beta_{0}\right)=m
$$

$4.3 \quad \frac{\partial n^{(0)}}{\partial \alpha_{0}} \neq 0, \quad \neq 0, \quad(2 n+1) w=k \pi$

$$
\mathrm{h}_{2 \mathrm{n}+1}\left(\alpha_{0}, \beta_{0}\right) \neq 0, \quad(2 \mathrm{n}+1) \mathrm{n}^{(0)}\left(\alpha_{0}, \beta_{0}\right)=\mathrm{m}
$$

4.4

$$
\begin{aligned}
& \frac{\partial \mathrm{n}^{(0)}}{\partial \alpha_{0}} \neq 0, \quad \neq 0, \quad(2 \mathrm{n}+1) \mathrm{w}=\mathrm{k \pi} \\
& \tau_{2(\mathrm{n}+1)}\left(\alpha_{0}, \beta_{0}\right) \neq 0, \quad 2(\mathrm{n}+1) \mathrm{n}^{(0)}\left(\alpha_{0}, \beta_{0}\right)=\mathrm{m}
\end{aligned}
$$

4. The conditions 4.1 and 4.2 have allowed to establ ish rather readily the existence of plane periodic motions of a nonspherical satellite in an elliptic orbit/in the vicinity of a circular synchronous motion/in a central gravity field. Periodic solutions were found in the cases of commensurability of the main frequence $n^{(0)}$ and of the mean orbital motion $\Omega$ of the form $n^{(0)}=\frac{\Omega}{3}, n^{(0)}=\frac{2}{3} \quad \Omega$ and $n^{(0)}=\frac{1}{4} \Omega$. To these solutions correspond the following generating values of the eccentricity of the satellite's orbit $e=e_{0}$ and of the dynamic compression $\alpha=\alpha_{0}$ : 


$$
e_{0}=0, \alpha_{0}=\frac{1}{9} ; e_{0}=0, \quad \alpha_{0}=\frac{4}{9} ; \quad e_{0}=0, \quad \alpha_{0}=\frac{1}{16}
$$

To these values of the parameters, correspond nonsymmetric periodic solutions.

The existence of symmetric periodic solutions, determined by the conditions $4.3,4.4$ in dependence of the type of resonance was also establ ished from the coefficients of the normal form of the familtonian of the problem; these Hamiltonians were constructed in [6].

5. The results obtained in $\$ \$ 1-3$ are valid for an investigation of periodic solutions of schwarzschild type of twodegree autonomous Hemiltonian systems. For this purpose, it is sufficient to $u$ se their reduction to a system of canonical equations with one and a half degrees of freedom, $1 . e$. to the equations of the form (1).

The same approach allowed to obtain constructive conditions of existence of periodic solutions in the vicinities of stationary solutions of two-degree autonomous lamiltonian systems, in particular, for the resonances $0: 1,1: 1,1: 3,1: 2$, etc. The conditions of existence of these periodic solutions were written down by means of expressions for frequencies and coefficients of normal forms of the Hamiltonians.

The results obtained allowed to study periodic solutions in the problem of motion of a point satellite in the equatorial plane of a rotating nonspherical planet. To these solutions correspond satellite's periodic motions in the vicinity of the planet's 1 ibration points. Planet's dynamic compressions $\alpha, \beta$ were used as active/varied/ parameters of the problem. As a result, symmetric period solutions were found in the vicinity of resonant curves $f \mathrm{~m} / \mathrm{n}\left(\alpha_{0}, \beta_{0}\right)=0$, corresponding to the commensurabilitys of the frequencies of the probl em $M: n=1: 2,1: 3$, as well as nonsymmetric periodic solutions in the vicinities of some particular points of these resonant curves.

By means of the above-mentioned conditions, was proved also the existence of periodic solutions in the restricted circular problem of three point bodies. To this solution correspond periodic motions of a point with a negligible mass in the vicinity of the triangular libration point by the commensurability of frequencies. Farlier, these motions were studied by means of numerical and analytical methods in the works of Henrard, Schmidt, et al. [7].

6. The restricted space of this report does not permit to consider in detail similar problems of investigation of periodic solutions of multidimensional Hamiltonian systems. Among the works of this research branch, devoted to a study of 
existence, construction, and stability of periodic solutions of miltonian systems in particular and degenerate cases /including the studies with an active use of the problem parameters/ are the works [2],[9],[10] etc.

These same works in fact indicate pathways and approaches to a more complete and thorough investigation of the periodicity conditions and of the periodic solutions themselves in a large variety of degenerate cases, in particular, to a study of periodic solutions of autonomous and nonautonomous Hamiltonian systems of the second, third and arbitrary orders in the vicinities of stationary and periodic reference solutions. It seens thereby possible to use efficiently the results of construction of the Hamiltonian's normal forms both for genera1-form sy stems and for specific problems of Celestial Mechanics, stated in [5],[11], etc.

7. By means of the H.Poincaré's classical conditions and of the above-mentioned constructive elaboration, a systematic study s done of periodic solutions in the plane and spatial problems of the motion of two mutually gravitating bodies, which either possess quasiconcentric density distributions or are represented by homogeneous ellipsoids with small compressions. Here new families of symmetric and nonsymmetric periodic solutions were found both in general and in degenerate cases, with an application of the procedure of the problem parameters' fitting, in the nonrestricted as well as in the restricted statements of the problem.

For these solutions, the motions of the centres of masses are close to the Bulerian ones of the bodies with spherical ellipsoids of inertia. In a few orbital revolutions, each body commits an integer number of revolutions around its own centre of masses.[10].

The existence vas studied of periodic solutions in the planetary versions of the problems of three and $n$ rigid bodies with small dimensions and with the ellipsoids of inertia close to spheres. These solutions generalize the H.Poincaré's classical periodic solution in the problem of three point bodies to a planetary sys tem of nonspherical rigid bodies [10][12].

An applicati on is presented of the found periodic solutions to a study of resonant phenomena in the motions of $t$ he Moon, Mercury, and Venus. It is shown, in particular, that the observed in the Venus' motion resonance is well described by one of the found first-kind periodic solutions in a nonrestricted three-body problem /Venus being a nonspherical rigid body, and the sun and the Earth - point masses/, with the necessary stability conditions fulfilled for this solution $[10]$. 


\section{APPEND IX}

Expansion for $z$ and formulae for $z_{1}^{(j)}, z_{2}^{(j)}$.

$$
\begin{aligned}
& z=z_{1}^{(j)} \mu^{\frac{1}{k_{j}(z)}}+z_{2}^{(j)} \mu^{\frac{2}{k_{j}(z)}}+\ldots, \\
& j=1 ; k_{1}(z)=1 \\
& z_{1}^{(j)}=-\psi_{1}\left(\frac{\partial \psi_{0}}{\partial z}\right)^{-1}, z_{2}^{(j)}=-\left(\frac{1}{2} \frac{\partial^{2} \psi_{0}}{\partial z^{2}} z_{1}^{(j)^{2}}+\frac{\partial \psi_{1}}{\partial z} z_{1}^{(j)}+\psi_{2}\right)
\end{aligned}
$$$$
\times\left(\frac{\partial \psi_{0}}{\partial z}\right)^{-1}
$$

$$
\begin{aligned}
& j=2,3 ; k_{2}^{(z)}=k_{3}^{(z)}=1 \\
& z_{1}^{(j)}= \pm \sqrt{-2 \psi_{1}\left(\frac{\partial^{2} \psi_{o}}{\partial z^{2}}\right)^{-1}}, z_{2}^{(j)}=-\left(\frac{\partial \psi_{1}}{\partial z}+\frac{1}{6} \frac{\partial^{3} \psi_{o}}{\partial z^{3}}\right) \\
& \times\left(\frac{\partial^{2} \psi_{0}}{\partial z^{2}}\right)^{-1}
\end{aligned}
$$

$$
\begin{aligned}
& j=4,5 ; k_{4}^{(z)}=k_{5}^{(z)}=1 \\
& p_{2}\left(z_{1}^{(j)}\right)=\frac{1}{2} \frac{\partial^{2} \psi_{0}}{\partial z^{2}} z_{1}^{(j)^{2}}+\frac{\partial \psi_{1}}{\partial z} z_{1}^{(j)}+\psi_{2}=0, \\
& z_{2}^{(j)}=-P_{3}\left(\frac{\partial P_{2}}{\left.\partial z_{1}^{(j)}\right)^{-1}}, j=6,7 ; k_{6}^{(z)}=k_{7}^{(j)}=2\right. \\
& P_{2}\left(z_{1}^{(j)}\right)=0, \frac{\partial P_{2}}{\partial z_{1}^{(j)}}=\frac{\partial \psi_{1}}{\partial z}+\frac{\partial^{2} \psi_{0}}{\partial z^{2}} z_{1}^{(j)}=0, z_{2}^{(j)}=0, \\
& z_{3}^{(j)}=-P_{3}\left(\frac{1}{2} \frac{\partial^{2} \psi_{0}}{\partial z^{2}}\right)^{-1} \\
& j=8, k_{8}^{(z)}=3
\end{aligned}
$$




$$
\begin{aligned}
& v_{3}\left(z_{1}^{(j)}\right)=\frac{1}{6} \frac{\partial^{3} \psi_{0}}{\partial z^{3}} z_{1}^{(j)^{3}}+\psi_{1}=0, z_{2}^{(j)}=-v_{4}\left(\frac{\partial v_{3}}{\partial z_{1}^{(j)}}\right)^{-1} \\
& j=9,10 ; k_{9}^{(z)}=k_{10}^{(z)}=2 \\
& u_{3}\left(z_{1}^{(j)}\right)=z_{1}^{(j)}\left(\frac{1}{6} \frac{\partial^{3} \psi_{0}}{\partial z^{3}} z_{1}^{(j)^{2}}+\frac{\partial \psi_{1}}{\partial z}\right)=0, \\
& z_{2}^{(j)}=-u_{4}\left(\frac{\partial u_{3}}{\partial z_{1}^{(j)}}\right)^{-1} \\
& j=11 ; k_{11}^{(z)}=1 \\
& z_{1}^{(j)}=-\psi_{2}\left(\frac{\partial \psi_{1}}{\partial z}\right)^{-1}, \quad z_{2}^{(j)}=-P_{3}\left(\frac{\partial \psi_{1}}{\partial z}\right)^{-1} \\
& j=12 ; k_{12}^{(z)}=3 \\
& z_{1}^{(j)}=0, \quad z_{2}^{(j)}=\left[-\psi_{2}\left(\frac{1}{6} \frac{\partial^{3} \psi_{0}}{\partial z^{3}}\right)^{-1}\right]^{1 / 3} \\
& j=13,14,15 ; \quad k_{13}^{(z)}=k_{14}^{(z)}=k_{15}^{(z)}=1 \\
& P_{3}\left(z_{1}^{(j)}\right)=\frac{1}{6} \frac{\partial^{3} \psi_{0}}{\partial z^{3}} z_{1}^{(j)^{3}}+\frac{1}{2} \frac{\partial^{2} \psi_{1}}{\partial z^{2}} z_{1}^{(j)}+\frac{\partial \psi_{2}}{\partial z} z_{1}^{(j)}+\psi_{3}=0, \\
& z_{2}^{(j)}=-P_{4}\left(\frac{\partial P_{3}}{\partial z_{1}^{(j)}}\right)^{-1}
\end{aligned}
$$$$
\text { Polynomials } \mathrm{P}_{4}\left(\mathrm{z}_{1}^{(j)}\right), \mathrm{v}_{4}\left(\mathrm{z}_{1}^{(j)}\right), \mathrm{u}_{4}\left(\mathrm{z}_{1}^{(j)}\right)
$$$$
\begin{aligned}
P_{4}\left(z_{1}^{(j)}\right)=\frac{1}{4 !} \frac{\partial^{4} \psi_{0}}{\partial z^{2}} z_{1}^{(j)^{4}} & +\frac{1}{3 !} \frac{\partial^{3} \psi_{1}}{\partial z^{3}} z_{1}^{(j)^{3}}+\frac{1}{2 !} \frac{\partial^{2} \psi_{2}}{\partial z^{2}} z_{1}^{(j)^{2}} \\
& +\frac{\partial \psi_{3}}{\partial z} z_{1}^{(j)}+\psi_{4}
\end{aligned}
$$ 
$v_{4}\left(z_{1}^{(j)}\right)=\frac{1}{4} ! \frac{\partial^{4} \psi_{0}}{\partial z^{4}} z_{1}^{(j)^{4}}+\frac{\partial \psi_{1}}{\partial z} z_{1}^{(j)}+\psi_{2}$

$u_{4}\left(z_{1}^{(j)}\right)=\frac{1}{4 !} \frac{\partial^{4} \psi_{0}}{\partial z^{4}} z_{1}^{(j)^{4}}+\frac{1}{2 !} \frac{\partial^{2} \psi_{1}}{\partial z^{2}} z_{1}^{(j)^{2}}+\psi_{2}$

2. Expressions for the functions $F_{i}^{(z, j)}, i=0,1,2$.

For $j=1,4,5,11,13,14,15$ :

$F_{o}^{(z, j)}=I_{1}, \quad F_{1}^{(z, j)}=\frac{\partial I_{1}}{\partial z} z_{1}^{(j)}+I_{2}$,

$F_{2}^{(z, j)}=\frac{\partial I}{\partial z} z_{2}^{(j)}+\frac{1}{\overline{2}} \frac{\partial^{2} I_{1}}{\partial z^{2}} z_{1}^{(j)^{2}}+\frac{\partial I_{2}}{\partial z} z_{1}^{(j)}+I_{3} \cdot$

For $j=2,3,6,7,9,10$ :

$F_{0}^{(z, j)}=I_{1}, \quad F_{1}^{(z, j)}=\frac{\partial I_{1}}{\partial z} z_{1}^{(j)}$,

$F_{2}^{(z, j)}=\frac{\partial I_{1}}{\partial z} z_{2}^{(j)}+\frac{1}{2} \frac{\partial^{2} I_{1}}{\partial z^{2}} z_{1}^{(j)^{2}}+I_{2}$.

For $j=8,12$ :

$F_{0}^{(z, j)}=I_{1}, \quad F_{1}^{(z, j)}=\frac{\partial L_{1}}{\partial z} z_{1}^{(j)}$,

$F_{2}^{(z, j)}=\frac{\partial I_{1}}{\partial z} z_{2}^{(j)}+\frac{1}{2} \frac{\partial^{2} I_{1}}{\partial z^{2}} z_{1}^{(j)^{2}}$.

3. Expressions for $\psi_{i}, I_{i}(i=0,1,2)$

$$
\begin{aligned}
& \psi_{0}=-T\left(\frac{\partial \mathrm{H}_{\mathrm{O}}}{\partial \mathrm{a}}+\frac{\mathrm{q}}{\mathrm{q}_{\mathrm{O}}} \Omega\right), \quad \mathrm{I}_{1}=\mathrm{T} \frac{\partial \overline{\mathrm{H}}_{1}}{\partial \mathrm{w}}, \\
& \psi_{1}=-\mathrm{T} \frac{\partial^{2} \mathrm{H}_{\mathrm{O}}}{\partial \mathrm{a}^{2}}\left(\frac{\partial \overline{\mathrm{H}}_{1}}{\partial \mathrm{w}}-\left.\int \frac{\partial \overline{\mathrm{H}}_{1}}{\partial \mathrm{w}} \mathrm{dt}\right|_{0}\right)-\frac{\partial \overline{\mathrm{H}}_{1}}{\partial \mathrm{a}} \mathrm{T},
\end{aligned}
$$




$$
\begin{aligned}
& \psi_{2}=-T\left(\frac{\partial^{2} H_{O}}{\partial \alpha^{2}} \overline{I_{2}(t)}+\frac{1}{2} \frac{\partial^{3} H_{O}}{\partial \alpha^{3}} \overline{\left(I_{1}(t)\right)^{2}}\right. \\
& \left.+\frac{\partial^{2} H_{1}}{\partial \underline{\alpha^{2}}} I_{1}+\frac{\partial^{2} \mathrm{H}_{1}}{\partial \alpha \partial w} \psi_{1}+\frac{\partial \bar{H}_{2}}{\partial \Phi}\right), \\
& I_{2}=T\left(\frac{\partial^{2} \mathrm{H}_{1}}{\partial \alpha \partial w} I_{1}+\frac{\overline{\partial^{2} H_{1}}}{\partial w^{2}} \psi_{1}+\frac{\partial \bar{H}_{2}}{\partial w}\right)
\end{aligned}
$$




\section{REFERENCES}

1 Poincare, H. New Methods in Celestial Mechanics. Selected Works. Nauka, Moscow, 1971, Vol. 1, p.7-326.

2 Barkin, Yu.V., Pankratov, A.A., Periodic solutions of Hamil tonian $s$ ystens and their application to satellites ' dynamics. Ko smicheskie issledovani ya. - 1986, Vol .24, No.3, p.345-357.

3 Deprit, A, Canonical transformations depending on a small parameter - Celestial Mechanics, 1969, Vol.1, No.1.

4 Hori, G.I., Theory of general perturbations with unspecified canonical variables, Publ. Astron. Soc.Japan, 1966, Vol. 18, No. 4, p.287-296.

5 Markeev, A.P., Libration points in Celestial Mechanics and co smodynamics, Nauka, Moscow, 1978, pp.312.

Zl atoustov, V.A., Markeev, A.P., Stability of planar oscillations of a satellite in an elliptic orbit, Celestial Mechanics, 1973, Vol.7, p.31-45.

Scrnidt, D.S., Periodic solutions near a resonant equilbrium of a Hamiltonian system, Celestial Mechanics,1974, Vol. 9, p.81- 103 .

Earkin, Yu. V., Const ructive methods of investigation of periodic solutions of multifrequency oscillatory systems and their applications to mechanics, All Union Conference on Nonlinear Oscillations of Mechanical Systems, Abstracts of papers, Gorky, 1987 .

9 Parkin, Yu. V., Pankratov, A.A., Periodic solutions of lamiltonian systems in some cases of degeneracy, Prikladna ya matematika i mekhanika, 1987, Vol. 51, No.2, p. 235-243.

10 Earkin, Yu, V., Dynamics of a system of nonspherical Celestial bodies and the theory of the Moon's rotation. Doctoral thesis. Moscow State University, Sternberg Astronomical Institute, $1989,412 \mathrm{pp}$.

11 Markeev, A.P., Medvedev, S.V., Sokol'skij, A.G., Met hods and algorithms for normalization of differential equations, Moscow Aviation Institute, 19,74 pp.

12 Barkin, Yu, V., Resonant motions of rigid bodies in the solar system, Dynamics of the Solar System/ Edited by M.Sidlichovsky, Proceedings of the Tenth European Reginal Astronomy Meeting of the IAU/Prague, 24-29 August, 1987/, Prague, 1987, Vol. 3, p.63-69. 